\title{
In vitro blood flow visualizations and cell-free layer (CFL) measurements in a microchannel network
}

\author{
D. Bento ${ }^{\mathrm{a}, \mathrm{b}}$, C.S. Fernandes ${ }^{\mathrm{b}}$, J.M. Miranda ${ }^{\mathrm{a}}$, R. Lima ${ }^{\mathrm{a}, \mathrm{c}, *}$ \\ ${ }^{a}$ CEFT, Faculdade de Engenharia da Universidade do Porto (FEUP), Rua Dr. Roberto Frias, 4200-465 Porto, Portugal \\ ${ }^{\mathrm{b}}$ Instituto Politécnico de Bragança, ESTiG/IPB, C. Sta. Apolónia, 5301-857 Bragança, Portugal \\ ${ }^{\mathrm{c}}$ MEtRiCS, Mechanical Eng. Dep., University of Minho, Campus de Azurém, 4800-058 Guimarães, Portugal
}

\section{A R T I C L E I N F O}

\section{Keywords:}

Microchannel network

Cell-free layer

Blood flow

Microfluidics

Red blood cells

\begin{abstract}
A B S T R A C T
Microvascular networks are not simple straight microchannels but rather complex geometries composed by successive asymmetric divergent and convergent bifurcations. Despite the extensive research work in this field, still lack of knowledge about the blood flow behavior in microvascular networks. The current study applies the most current advanced visualization and microfabrication techniques to provide further insights into to the blood flow in network geometries. Hence, by using a high-speed video microscopy system, blood flow measurements and visualizations of the cell-free layer (CFL) were performed along a microchannel network composed by several divergent and convergent bifurcations. The inlet flow rate was kept constant whereas the hematocrit (Hct) and the depth of the geometry was changed in order to evaluate their effects into the CFL thickness. The results, show clearly that the Hct has a significant impact on the CFL thickness whereas the effect of reducing the depth did not contribute to a noticeable change on the CFL. In addition, the in vitro blood flow results reported here provide for the first time that in microfluidic devices having several asymmetric confluences it is likely to have the formation of several CFLs not only around the walls but also in middle of the main channels just downstream of the last confluence apex. Although, to best of our knowledge there is no evidence that this kind of flow phenomenon also happens in vivo, we believe that for microvascular networks with similar geometries and under similar flow conditions tested in this work, this kind of phenomenon may also happen in vivo. Furthermore, the results from this study could be extremely helpful to validate current numerical microvascular network models and to develop more realistic multiphase numerical models of blood flow in microcirculation.
\end{abstract}

\section{Introduction}

In recent years, biofluids research conducted with microfluidic devices has increased exponentially. These devices offer the potential of gaining insights on several fundamental blood flow phenomena that occurs at a microscale level. The complex characteristics of the blood flow in microcirculation are mainly due to the presence of red blood cells (RBCs), which constitute the majority of the blood cellular matter. The concentration of RBCs in plasma and their deformability, in healthy conditions, plays an important role to define blood as a non-Newtonian fluid [1]. Some diseases, such as sickle-cell anemia, malaria, diabetes, polycythemia, cancer, heart disease are responsible to change the rheological and flow properties of the blood [2-10].

In microfluidic devices, blood exhibits unique flow structures and rheological behaviors, such as a cell-free layer (CFL) around the walls, a $\mathrm{RBC}$-rich core in the center region, a blunt velocity profile, as well as the vessel size dependent effective viscosity (known as FahraeusLindqvist effect) $[2,6]$. By decreasing the tube diameter there is a decrease of the apparent viscosity, due to the migration of RBCs towards the core flow and consequent increase of the CFL thickness around the walls. These microscale hemodynamic phenomena play an important role in blood mass transport mechanisms $[3,11]$. The CFL thickness depends of several factors such as cell concentration, deformability, vessel diameter, cell aggregation, flow rate, presence of microbubbles and geometry of the microchannels [12-20]. For instance, by increasing the concentration of RBCs, i.e., increasing the hematocrit (Hct), there is a decrease of CFL thickness [17,21].

The bifurcations are geometries extremely common in both microvessels and microfluidic devices and it is important to improve our current understanding regarding the influence of the bifurcations and confluences on the blood flow behavior at a microscale level. As a result, several numerical and in vitro blood flow studies have investigated the effect these complex

\footnotetext{
* Corresponding author.

E-mail address: rl@dem.uminho.pt (R. Lima).
} 
geometries on the blood flow [12,22-28]. From the beginning of this century several in vitro blood flow phenomena were measured and visualized in microchannels. For instance, when a suspension of RBCs flows through an asymmetric bifurcation, the volume fraction of RBCs increases in the daughter branch with a higher flow rate, whereas the volume fraction of RBCs decreases in the daughter branch with a lower flow rate. This phenomenon is known as Zweifach-Fung effect or simply as bifurcation law $[17,26,29]$.

In bifurcation channels, the flow behavior and concentration of RBCs depend on several key factors such as the size and heterogeneous distribution of cells within the parent channel $[27,30,31]$, the flow rate ratio of daughter branches [25,29,32], the plasma viscosity [27,33], the hematocrit distribution [23,30,34-36] and the RBCs properties including deformability and aggregation of RBC [12,22,24,28]. In general, when blood flows through a divergent bifurcation, RBCs travel into different daughter branches with different Htc levels. The separation of the plasma from the cells can be related to several flow phenomena such as the non-uniform RBC distribution at the parent channel, the interactions between the neighboring RBCs and the deviation of RBC trajectories from the flow streamlines at the bifurcation region [26].

Recently Ishikawa et al. [37], Leble et al. [38] and Pinto et al. [39] have performed in vitro blood flow studies in simple microchannels with symmetric bifurcations and confluences. In those works, it was observed a cell-depleted layer not only around the microchannel walls but also at the region of the confluence apex. However, no work has yet investigated the CFL formation in more complex geometries such as in a microchannel network. To the best of our knowledge, this is the first in vitro blood study that shows the CFL formation and behavior along a microchannel network composed by several divergent and convergent bifurcations.

\section{Materials and methods}

\subsection{Geometry and flow conditions of the microchannel network}

Previous in vitro blood studies performed in simple symmetric bifurcations have observed CFLs around the confluence apex studies [37-39]. The present study aims, to investigate CFL formation in more complex geometries such as in a microchannel network composed by several divergent and convergent bifurcations. Hence, in this study the shape and angles of the microchannel network was selected not only by taking into account the past in vitro flow studies geometries but also the angles that can be frequently found in vivo microvascular networks [40,41]. Fig. 1 shows a general view and main dimensions of the geometry of the microchannel network. The microchannels used in this study are composed by several divergent bifurcations and convergent bifurcations (confluences) with two different heights, i.e., 100 and $50 \mu \mathrm{m}$.

According to several in vivo experiments [2,42,43], typical average shear rates values frequently observed in vivo microvessels can vary
Table 1

Average shear rates $(\dot{\gamma})$ for each branch of the proposed microchannel network for a flow rate of $60 \mu \mathrm{l} / \mathrm{min}$.

\begin{tabular}{lll}
\hline Region & $\dot{\gamma}\left(\mathrm{s}^{-1}\right) 100 \mu \mathrm{m}$ height & $\dot{\gamma}\left(\mathrm{s}^{-1}\right) 50 \mu \mathrm{m}$ height \\
\hline A1 & 375.00 & 1250.00 \\
A2 & 374.99 & 1250.00 \\
B1 & 543.62 & 1656.15 \\
B2 & 558.36 & 1620.64 \\
C1 & 398.02 & 1186.56 \\
C2 & 389.21 & 1157.87 \\
D1 & 989.62 & 2453.89 \\
D2 & 991.49 & 2331.37 \\
E1 & 813.21 & 2106.60 \\
E2 & 858.68 & 2164.20 \\
E3 & 982.19 & 2538.98 \\
E4 & 980.49 & 2458.38 \\
F1 & 449.97 & 1405.27 \\
F2 & 444.82 & 1308.31 \\
\hline
\end{tabular}

from $200 \mathrm{~s}^{-1}$ up to $2000 \mathrm{~s}^{-1}$. Hence, before the network fabrication, CFD studies (using a commercial software Ansys, see supplementary material), were performed by assuming a Newtonian rheology and a flow rate of $60 \mu \mathrm{l} / \mathrm{min}$. Table 1 shows the estimated average or pseudo shear rates ( $\dot{\gamma}=$ average velocity/hydraulic diameter) for each branch of the proposed microchannel network. By analyzing the estimated average shear rates of the proposed network, it was observed that the majority of the calculated shear rates are among the typical values frequently observed in vivo microvessels, such as arterioles, capillaries and venules $[2,42,43]$. Hence, the proposed network geometry combined with a flow rate of $60 \mu \mathrm{l} / \mathrm{min}$ is representative of flow conditions happening in microcirculation.

\subsection{Experimental set-up}

All experiments were conducted by using a high-speed video microscopy system. The main equipment of this system consists of an inverted microscope (IX71, Olympus, Japan) and a high-speed camera (Fastcam SA3, Photron, USA) (see Fig. 2). The microchannel network was fabricated by using a soft lithography method [44] and a syringe pump (PHD ULTRA, Harvard Apparatus, USA) was used to impose a constant pressure driven flow of the fluids.

All the blood samples used in the present work were obtained from a healthy donor. Note that, the tubes used to collect the blood contain ethylenediaminetetraacetic acid (EDTA) to prevent coagulation. Additionally, all the RBCs were washed and separated from the blood by centrifugation by using physiological saline. Finally, the RBCs were then re-suspended in Dextran 40 (Dx 40) to make up the required haematocrits (Hcts). All blood samples were stored hermetically at $4{ }^{\circ} \mathrm{C}$ until blood flow experiments were carried out. All experiments were

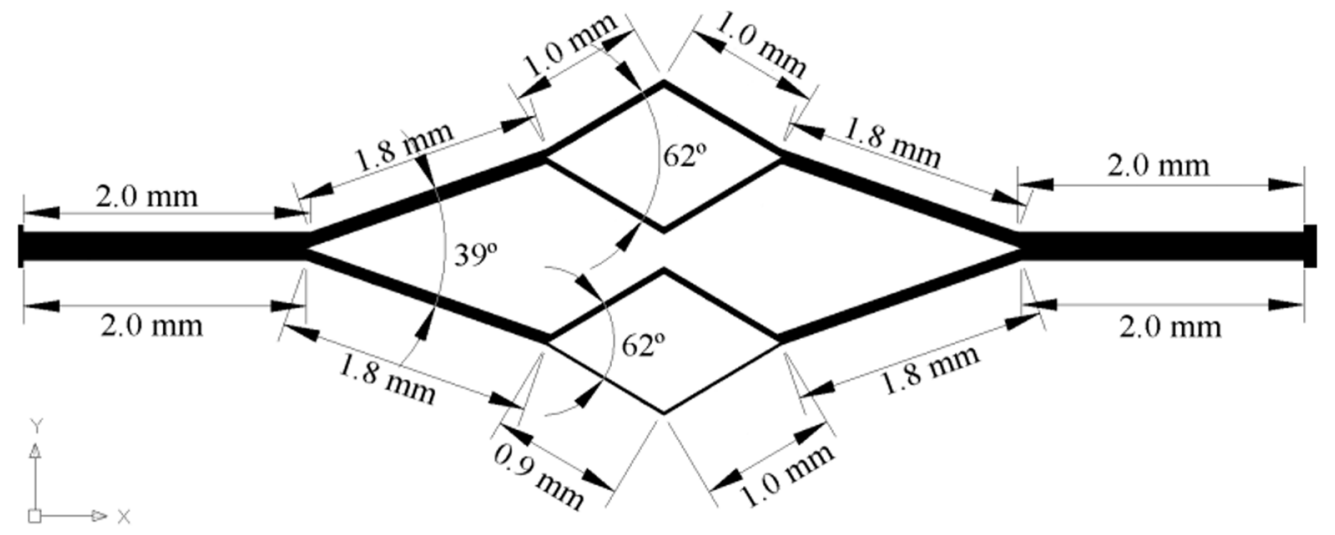

Fig. 1. The microchannel network geometry used to study the CFL behavior. 


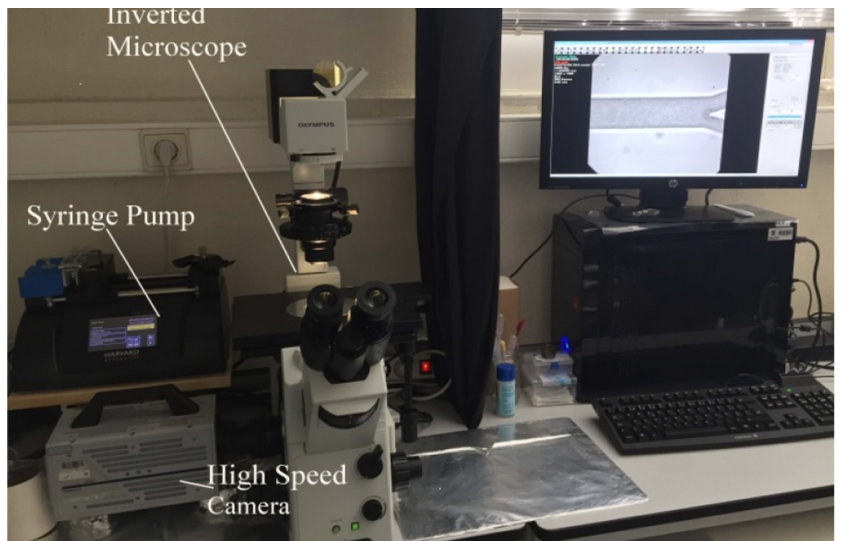

Fig. 2. High-speed video microscopy system composed by following equipment: inverted microscope, high-speed camera and syringe pump.

conducted at a room temperature of about $22^{\circ} \mathrm{C}$. In microcirculation, the Hct tends to decrease with the size of the microvessels. For instance, for a microvessel with a diameter around $25 \mu \mathrm{m}$ the Hct is around $12 \%$ [45]. Therefore, in this study, we have analysed the CFL for a flow rate of $60 \mu \mathrm{l} / \mathrm{min}$ for three working fluids with different Hcts, i. e., $5 \%$ Hct $10 \%$ Hct and $15 \%$ Hct.

\subsection{Image processing}

The images were obtained around the center plane of the microchannel, by using a frame rate of 2000 frames/second. All sets of frames were analyzed using the $\mathrm{Z}$ Project method from the ImageJ software [46]. The number of analyzed frames were about 400 for each movie. The recorded sequence of images was replaced by an image that represents the minimum intensity in each pixel. By using this methodology it is possible to distinguish two different regions with different intensities, i. e., the high intensity region corresponds to the CFL whereas the low intensity region corresponds to the RBCs core. In Fig. 3 it is possible to observe an image of the original movie (Fig. 3a)) and an image obtained by applying Z Project min intensity to all images of a movie (Fig. 3b)).

The pixel intensity profile was taken along lines perpendicular to the flow direction in several positions of the geometry network (see Fig. 4). By using this method, it is possible to identify the different regions where intensities are similar. The regions with the highest

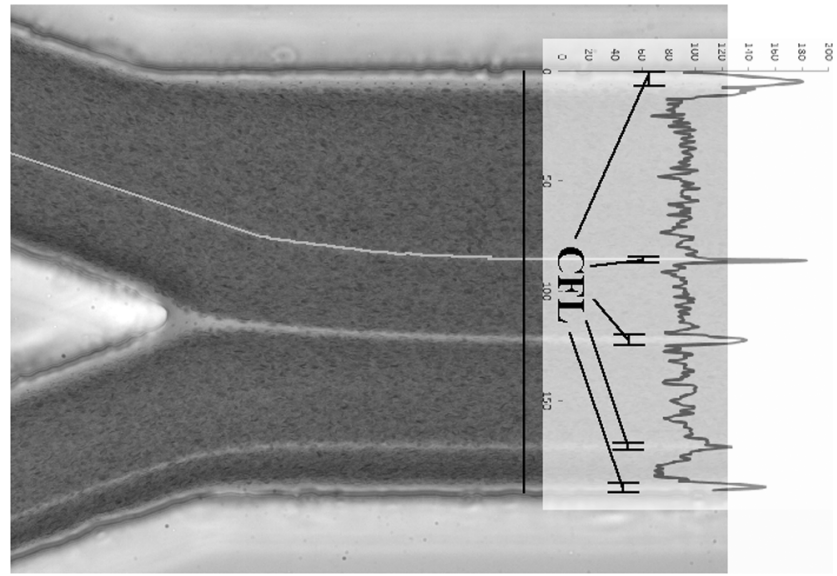

Fig. 4. Z project image and the correspondent profile of the pixel intensity.

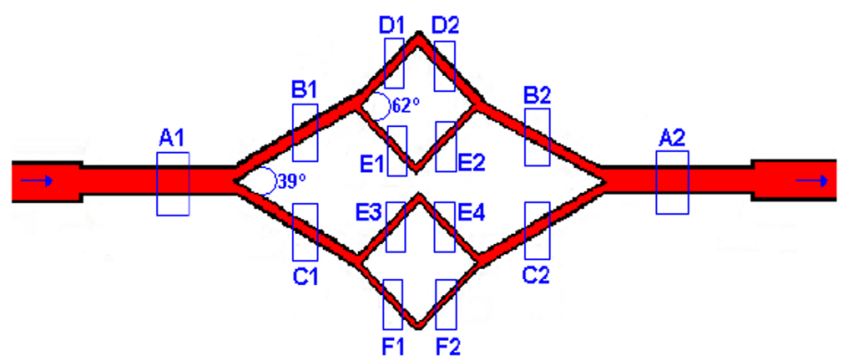

Fig. 5. Schematic diagram of the network geometry and the different regions analyzed in this study. The similar widths correspond to the same letter: $\mathrm{A} 1=\mathrm{A} 2=200 \mu \mathrm{m}, \mathrm{B} 1=\mathrm{B} 2=118.1 \mu \mathrm{m}, \mathrm{C} 1=\mathrm{C} 2=84.8 \mu \mathrm{m}, \mathrm{D} 1=\mathrm{D} 2=$ $58.19 \mu \mathrm{m}, \mathrm{E} 1=\mathrm{E} 2=\mathrm{E} 3=\mathrm{E} 4=46.55 \mu \mathrm{m}, \mathrm{F} 1=\mathrm{F} 2=23.29 \mu \mathrm{m}$.

intensities correspond to the CFL and the regions where intensities are smaller correspond to the RBCs. By measuring these regions in the diagram, it is possible to obtain the CFL thickness within the channels.

\section{Results and discussion}

After the application of the image processing method, the widths of the CFLs were measured. Different regions of the network, shown in the schematic diagram of Fig. 5, were studied. In Fig. 5, the channels of the geometry with the similar widths are labeled with the same letter, i. e.

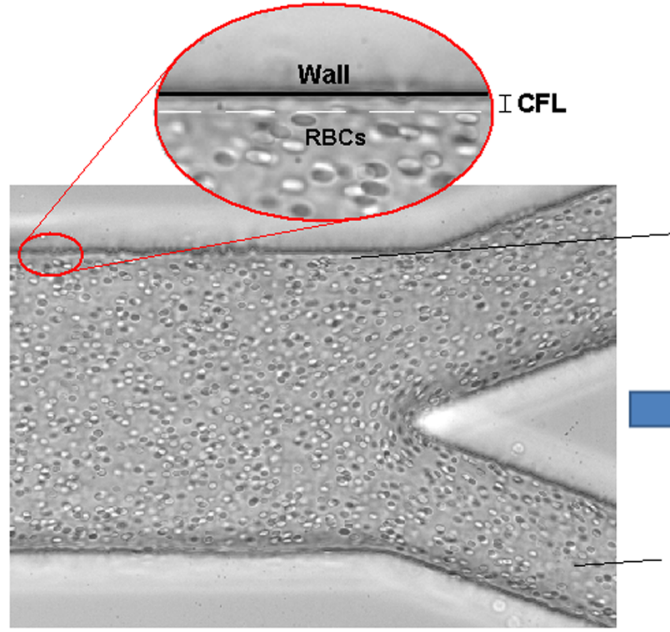

a)
CFL

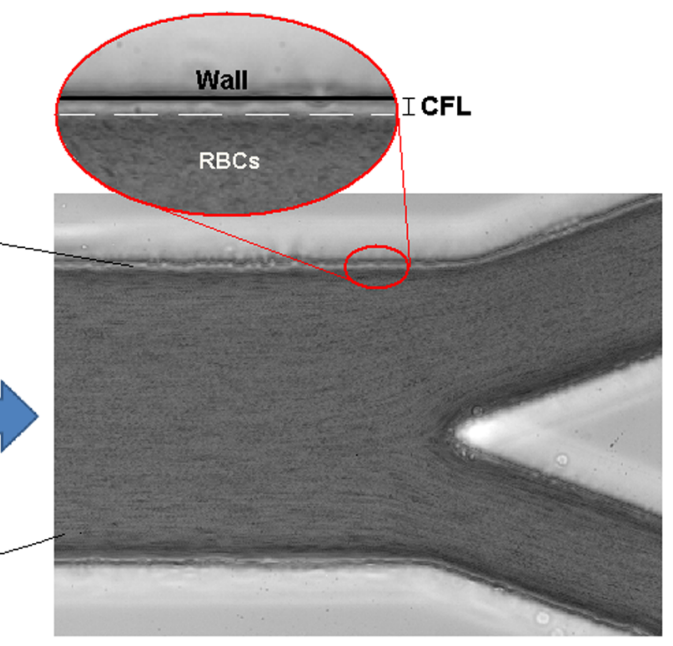

b)

Fig. 3. Z Project sequence image: (a) Image of the original movie (b) Z Project min intensity image. 


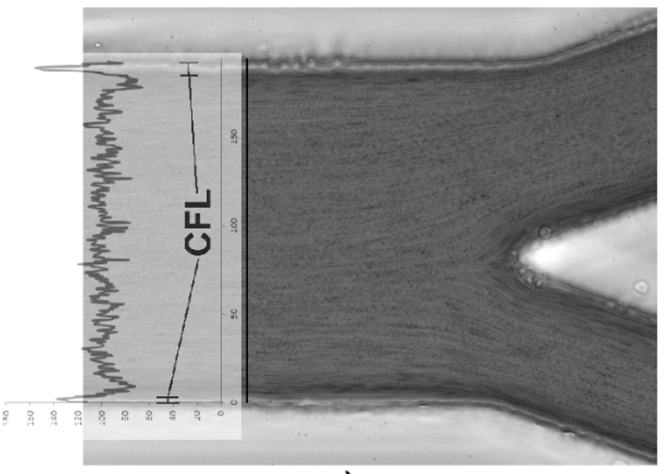

a)

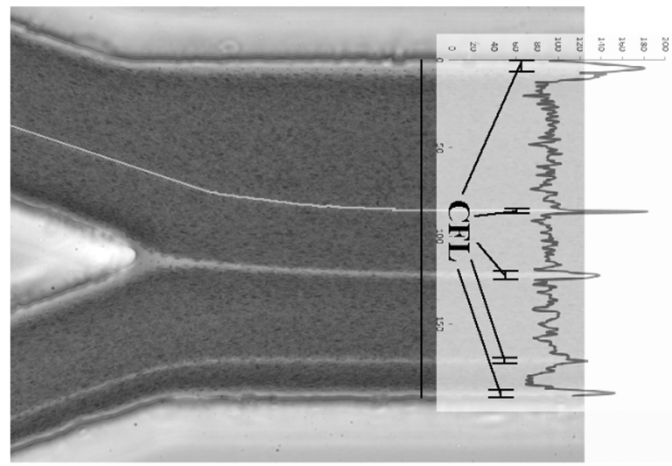

b)

Fig. 6. The different CFLs present at the a) inlet (A1) b) outlet (A2) of the network.
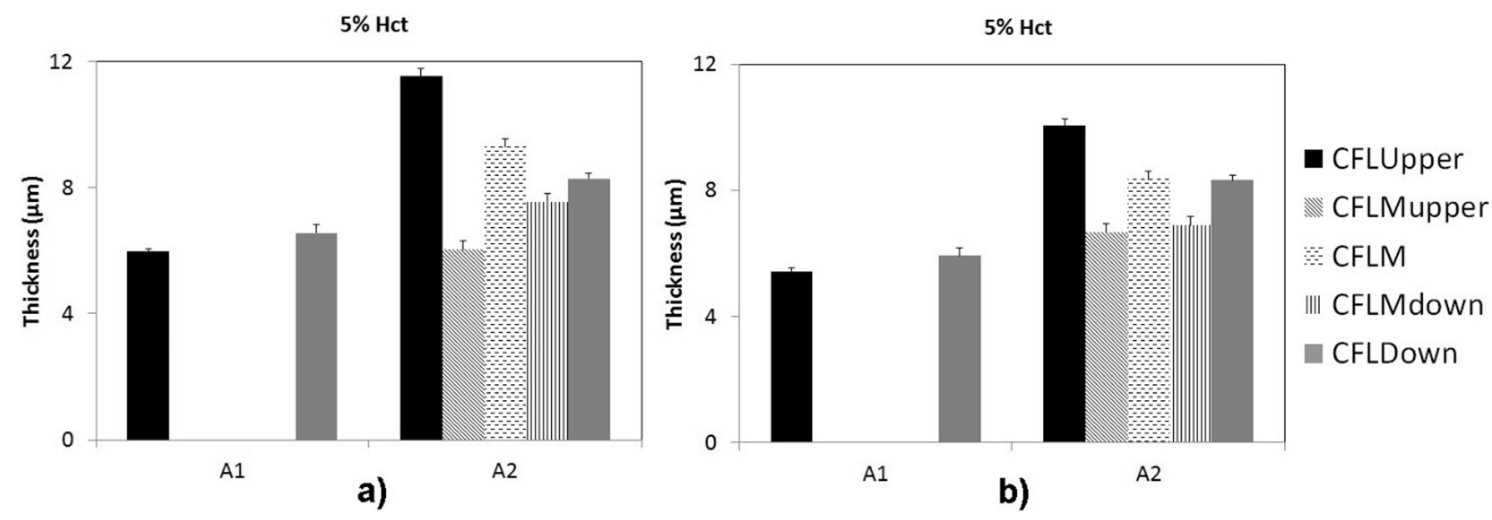

Fig. 7. CFL thickness at the inlet and outlet of the network for a Hct of $5 \%$ and at different heights: (a) $100 \mu \mathrm{m}$ and (b) $50 \mu \mathrm{m}$.

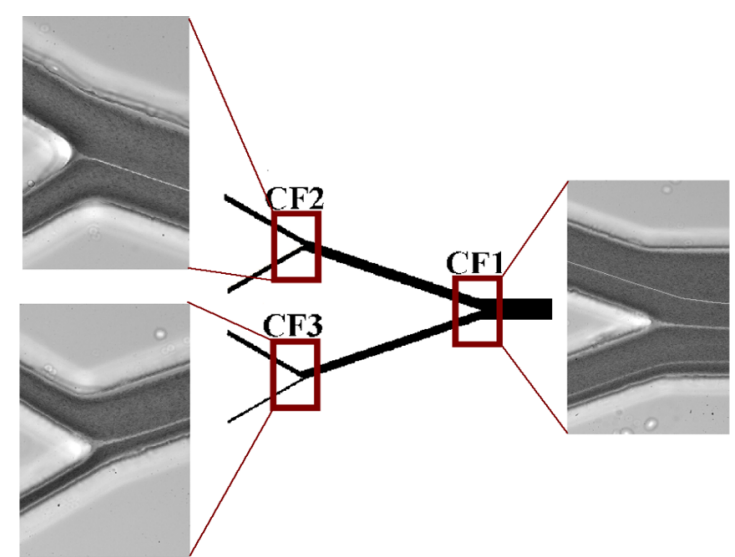

Fig. 8. The different CFLs present in each confluence.

A1 and A2 both have $200 \mu \mathrm{m}, \mathrm{B} 1$ and B2 both have $118.1 \mu \mathrm{m}$.

In order to understand the formation of the CFL and the overall influence of the bifurcations and confluences, firstly the inlet (A1) and outlet (A2) of the channel were analyzed. Fig. 6 shows the different CFLs present at the inlet (A1) and outlet (A2) of the network (see supplementary videos 1 and 2).

Fig. 7 shows the CFL thickness at the inlet and outlet of the tested network for a Hct of $5 \%$ and a height of $100 \mu \mathrm{m}$ and $50 \mu \mathrm{m}$. At the first bifurcation located at the entrance (A1), it is clear the presence of only two CFLs along the channel walls, whereas at the exit (A2) in addition to the CFLs around the walls there is the presence of 3 new CFLs located at the center of the outlet channel (A2). These new CFLs are a consequence of the effect of the confluence vertices that are present in this network geometry. For each convergent bifurcation, the cell-depleted layer formed around the inner walls promotes the formation of a CFL immediately downstream of the confluence apex. These thin CFLs formed at the center of the channel remains along the network and due to the presence of 3 confluences in this network at the end it is possible to observe 3 new CFLs at the channel outlet. These results corroborate past results performed in simple confluence geometries [37-39]. In Fig. 8 we can see the different CFLs present in each confluence.

In Fig. 9, it is shown the thickness of the CFLs at the different confluences (CF1, CF2, CF3) for the different hematocrits. Note that, the depth of this network geometry was around $100 \mu \mathrm{m}$ along the full length. In these data, it is clear the formation of a CFL in the apex of the different confluences (CF1, CF2, CF3). Additionally, by increasing the Hct there is a decrease in the thickness of all existing CFLs. This latter phenomenon is in accordance with the previous results observed in both in vivo and in vitro experiments [20,39,47-49].

For the network geometry with a depth of $100 \mu \mathrm{m}$, the results indicate that the CFL thickness formed at the confluence apex (CFLM) tend to increase along the network. For instance, the CFLM at CF1 is always the highest for all the tested Hcts. In addition, by comparing the CFL thickness at CF2 and CF3, the results show that the CFL at CF3 tend to be higher than CF2. This result can be explained by the bifurcation law as in asymmetric bifurcations the Hct is superior in the channels where the flow rate is larger, making the cell-free region higher in the smaller channels. This effect is more evident for low Hct, i. e., 5\% Hct.

Overall, the CFL at the CF1 region is the highest for all the tested Hcts. This is more evident for the CFL at upper part (CFLupper) and at middle of the channel (CFLM). This CFL increase was observed for different Hcts and as a result this phenomenon is mainly due to the geometry of microchannel network. These results suggest that the dimensions of the branches and abrupt changes of the flow direction play a major role on the CFL increase. The present study shows clearly that the higher dimensions of the branches at the upper part of the network (CF2) increase both flow rate and mechanical stresses and as a result might be the main cause for the highest CFL observed at upper part of 


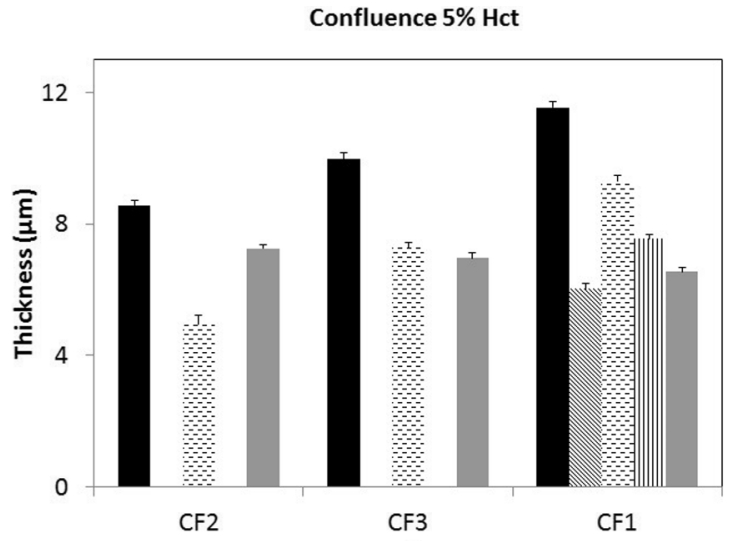

a)

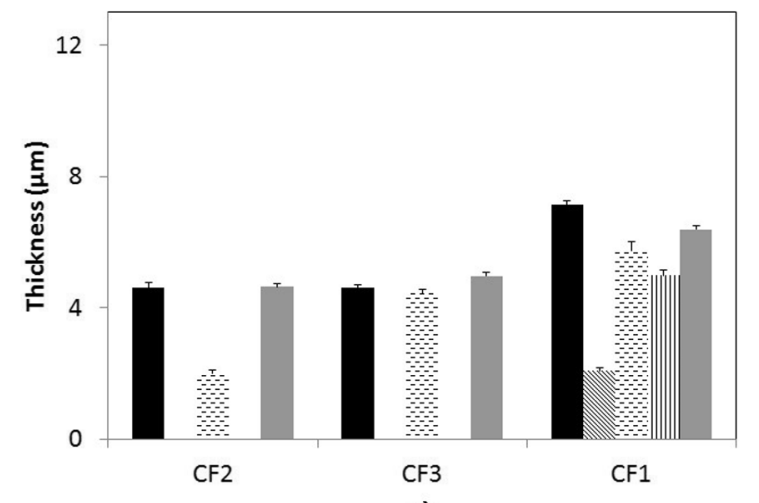

c)

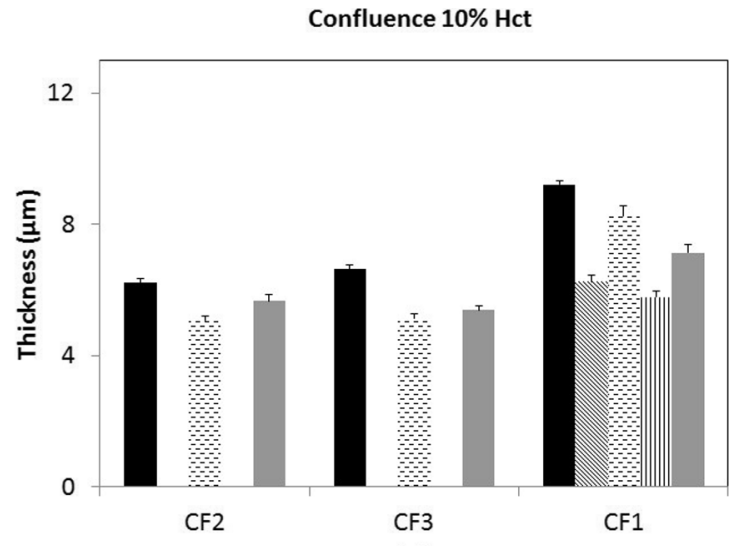

b)
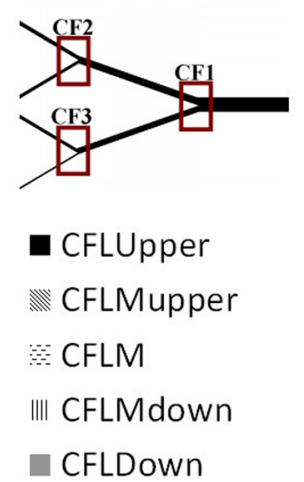

CFLDown

Fig. 9. CFL thickness in CF1, CF2 and CF3 in geometry with $100 \mu \mathrm{m}$ of height with (a) $5 \%$ Hct, (b) $10 \%$ Hct and (c) $15 \%$ Hct.
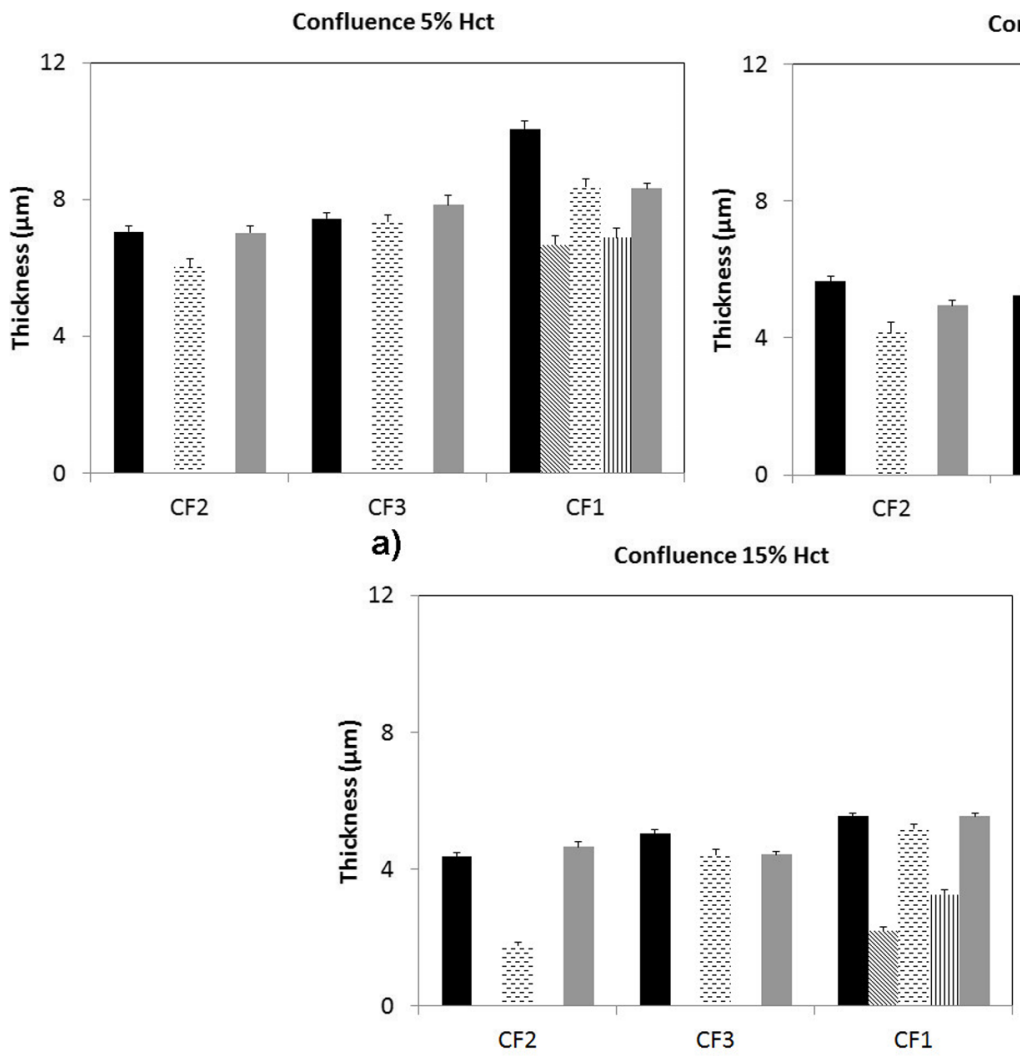

c)

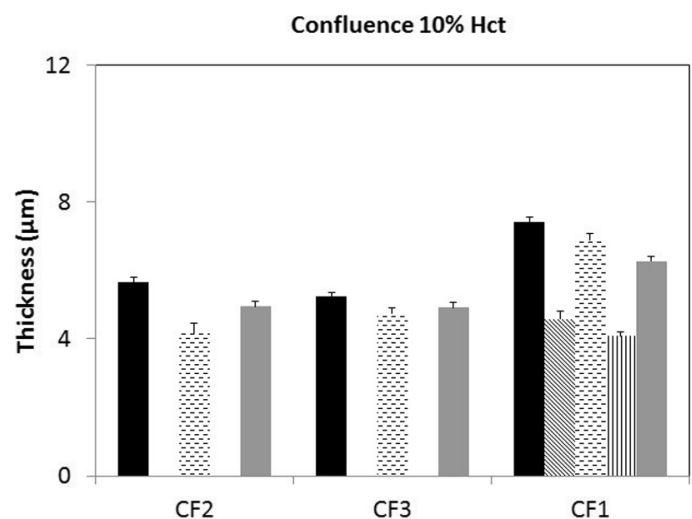

b)

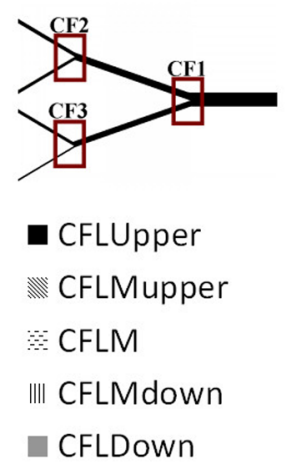

Fig. 10. CFL thickness in CF1, CF2 and CF3 in geometry with $50 \mu \mathrm{m}$ of height with (a) $5 \%$ Hct, (b) $10 \%$ Hct and (c) $15 \%$ Hct. 


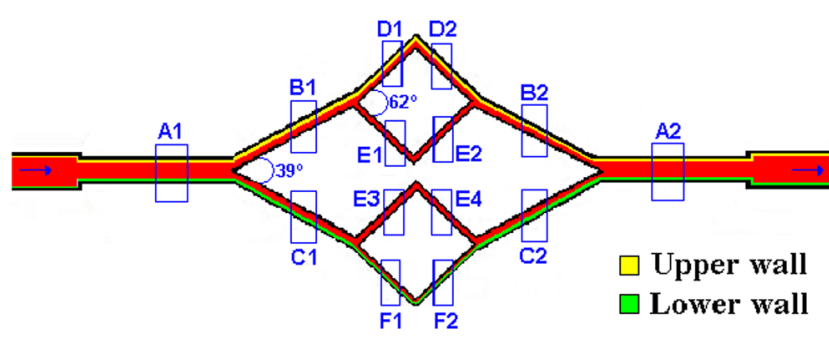

Fig. 11. Schematic diagram of network geometry and the CFLs thickness around the upper and lower walls.

the CF1 region. However, it is important to point out that the effect of the angles on the CFL thickness requires further investigation by performing similar blood flow studies in both simple and complex networks but this time by only changing the angles in a meaningful way. An adequate alternative to study such effect is by using as reference a geometry that follows the Murray law [50-52].

Another interesting result observed downstream of the apex CF1 (A2) is the formation of 3 CFLs in middle of the microchannel. Besides the CFL formed at CF1, the other two were formed at CF2 and CF3 and they remain visible along the geometry due to laminar flow that is the most often regime in microcirculation where viscous force dominates over inertial forces. These blood flow results provide a strong evidence that in microfluidic devices with convergent bifurcations it is likely to have the formation of CFL not only around the walls but also in middle of the microchannels. Although, to best of our knowledge there is no evidence that this kind of flow phenomena also happens in vivo, we believe that with the progress of the visualizations techniques and numerical models it will be possible to make this invisible phenomenon to be visible in microvessels.

In addition, in this work, we can show that there is a clear influence of both dimensions of the branches and asymmetry of the geometry on the location of the CFL immediately downstream of the final confluence apex. This effect can be clearly seen in Figs. 6 and 8. Previous studies performed with a symmetric network have shown that location of the CFL downstream the confluence apex is around the center of channel $[38,39]$. However, from this present study it is clear that the CFL formed at the final confluence suffers a small deviation and is no longer in the center of the channel (see supplementary video 2).

To evaluate the effect of the depth, Fig. 10 shows the CFL thickness at different confluences for the same network geometry but this time with the height of $50 \mu \mathrm{m}$. Overall, it is possible to observe that the CFL tends to decrease as the height reduces. This effect is more evident at the CF1 region. In addition, these results corroborate qualitatively the previous ones where not only the CFL decreases with the increase of the Hct but also there is a clear formation of 3 CFLs in middle of the microchannel located downstream of the apex CF. It is also possible to observe that qualitatively the CFL thickness tend to increase along the full length of the network geometry.

To obtain further insights into the behavior of the CFL around the walls, the CFL thickness was measured along the network geometry at both upper and lower walls (see Fig. 11).

Fig. 12, shows that, for both networks ( $50 \mu \mathrm{m}$ and $100 \mu \mathrm{m}$ depth) and different Hcts tested in this study, the behavior of the CFL thickness along the geometry is similar at both upper and lower wall. These results show that the thickness of the CFL decreases as the channel width decreases reaching a minimum value at the region where there is an abrupt change of the flow direction (D1/D2; F1/F2). After passing this bend the CFL starts to increase again reaching a maximum value at the highest dimension of the network located at the region A2. The influence of the bend (D1/D2) and other geometrical effects on the CFL thickness can be clearly observed at Fig. 13.

Fig. 13 shows the trajectory of a representative RBC flowing around the CFL boundary at the upper wall of a network microchannel with the height of $100 \mu \mathrm{m}$. In Fig. 13a, it is possible to observe that there is a clear CFL increase due change of the flow direction without a change in the width of the channel. In Fig. 13a and b, it is also clear an increase of the CFL thickness due bends but this time the channels widths also increase and consequently play a major role into the increase of the CFL thickness. Hence, the combination of these geometrical effects provide a strong evidence about the observed increased of the CFL in this kind of microchannel network.
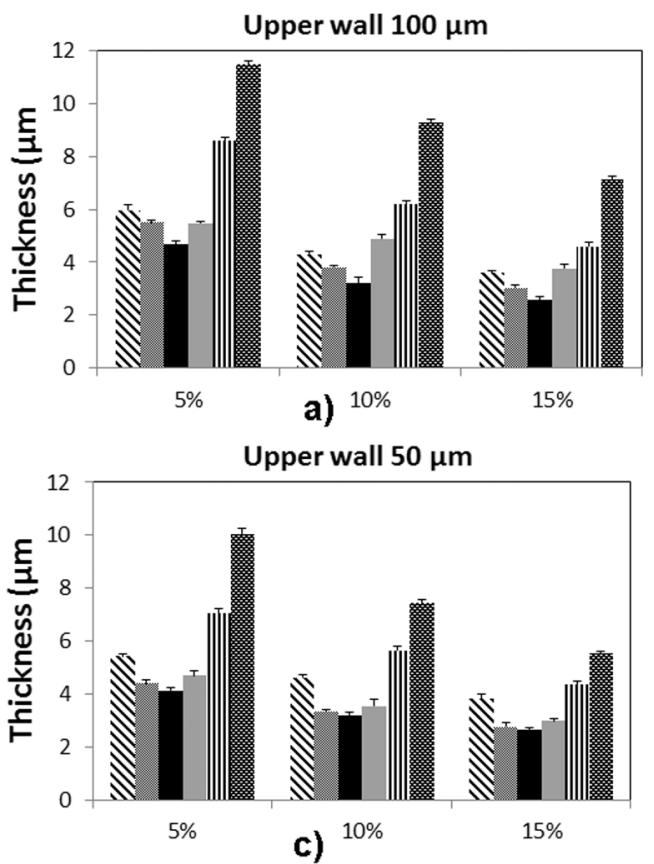
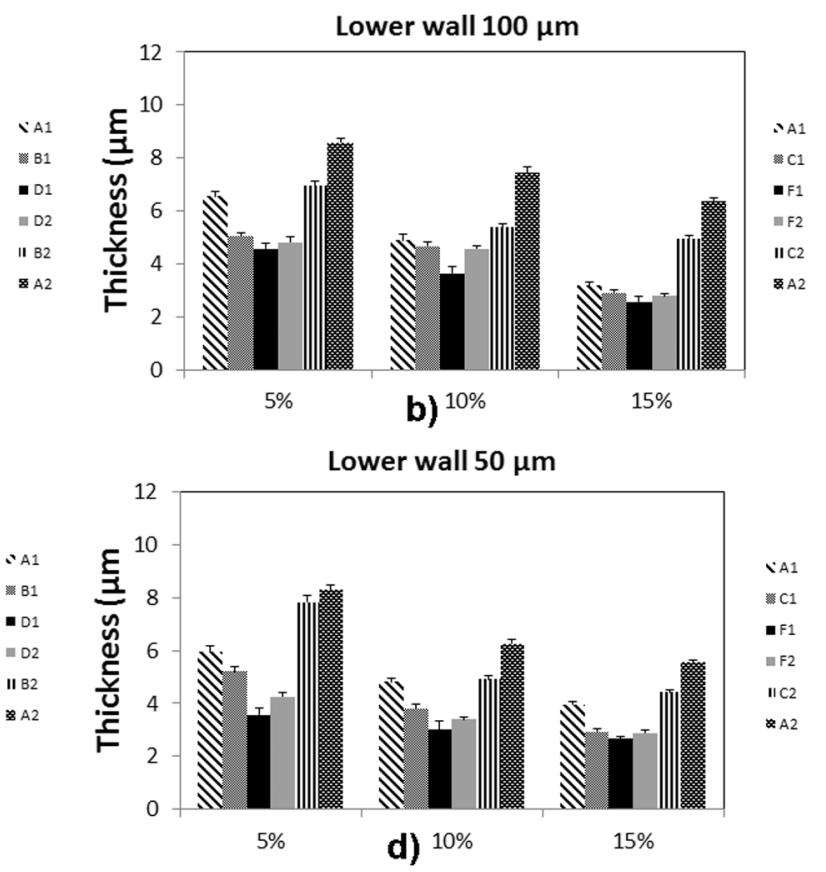

Fig. 12. CFL thickness behavior in (a) upper wall (b) lower wall in geometry with $100 \mu \mathrm{m}$ of height, and (c) upper wall (d) lower wall in geometry with $50 \mu \mathrm{m}$ of height. 


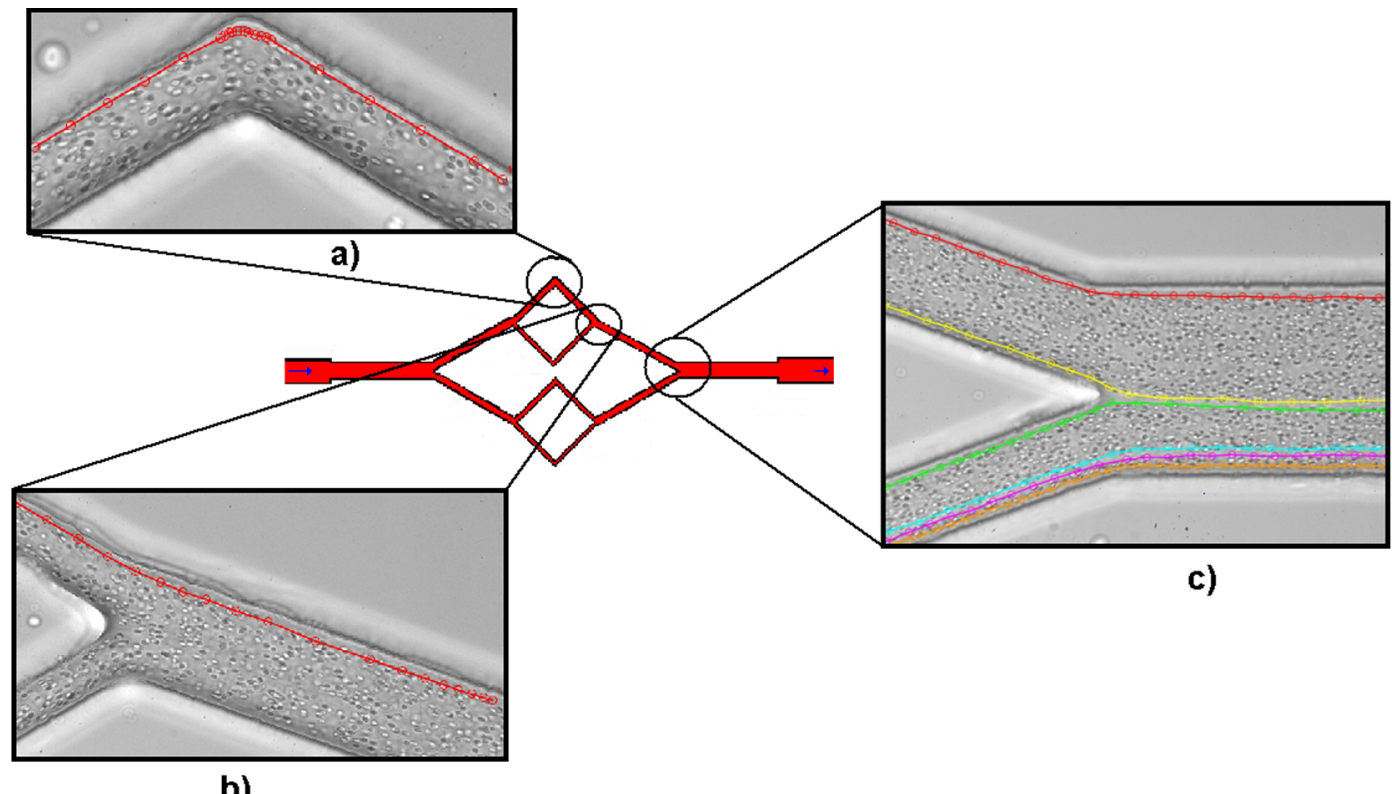

b)

Fig. 13. Representative RBC trajectory flowing around the CFL boundary at the upper wall of a network microchannel with the height of $100 \mu \mathrm{m}$ : (a) bend; (b) CF2, and (c) CF1.

\section{Conclusions}

The present study shows in vitro blood measurements and visualizations of the CFL formation and behavior along a microchannel network composed by several divergent and convergent bifurcations. During all the experiments, the flow rate was kept constant whereas the Hct and depth of the geometry was changed to evaluate their effect in the CFL thickness. Comparing all the obtained the results, it is clear that the Hct has significant impact on the CFL thickness whereas the effect of reducing the depth did not contribute to a noticeable change on the CFL. In fact, the results acquired from this study, show clearly that regions located at the microchannel network that promote abrupt flow directions plays an important role in rising the CFL thickness along the network.

In addition, the in vitro blood flow results reported here provide for the first time that in microfluidic devices having several asymmetric confluences it is likely to have the formation of several CFLs not only around the walls but also in middle of the main channels just downstream of the last confluence apex. Although, to best of our knowledge there is no evidence that this kind of flow phenomena also happens in vivo, we believe that with the progress of the flow visualization techniques and numerical models it will be possible to make this invisible phenomenon to be visible in microvessels.

\section{Declaration of Competing Interest}

The authors have no conflict of interest to report.

\section{Acknowledgments}

This work was supported by Fundação para a Ciência e a Tecnologia (FCT), Portugal, under the strategic grants UID/EMS/04077/2019, UID/EEA/04436/2019 and UID/EMS/00532/2019. The authors are also grateful for the partial funding of FCT through the projects POCI01-0145-FEDER-016861, POCI-01-0145-FEDER-028159, NORTE-010145-FEDER-029394, NORTE-01-0145-FEDER-030171, funded by COMPETE2020, NORTE2020, PORTUGAL2020, and FEDER, and the PhD grant SFRH/BD/91192/2012.

\section{Appendix A. Supplementary material}

Supplementary data to this article can be found online at https:// doi.org/10.1016/j.expthermflusci.2019.109847.

\section{References}

[1] E. Kaliviotis, et al., Red blood cell aggregate flux in a bifurcating microchannel, Med. Eng. Phys. 48 (2017) 23-30.

[2] A.S. Popel, P.C. Johnson, Microcirculation and hemorheology, Annu. Rev. Fluid Mech. 37 (2005) 43-69.

[3] Dmitry A. Fedosov, B. Caswell, Aleksander S. Popel, George Em Karniadakis, Blood flow and cell-free layer in microvessels, Microcirculation 17 (2010) 615-628.

[4] D. Fedosov, et al., Quantifying the biophysical characteristics of Plasmodium-falciparum-parasitized red blood cells in microcirculation, Proc. Natl. Acad. Sci. 108 (1) (2011) 35-39.

[5] V.K. Katiyar, D. Fisseha, Analysis of mechanical behavior of red blood cell membrane with malaria infection, World J. Mech. 1 (03) (2011) 100.

[6] X. Yin, T. Thomas, J. Zhang, Multiple red blood cell flows through microvascular bifurcations: cell free layer, cell trajectory, and hematocrit separation, Microvasc. Res. 89 (2013) 47-56.

[7] Z. Shen, et al., Inversion of hematocrit partition at microfluidic bifurcations, Microvasc. Res. 105 (2016) 40-46.

[8] G. Tomaiuolo, et al., Microconfined flow behavior of red blood cells, Med. Eng. Phys. 38 (1) (2016) 11-16.

[9] D. Bento, et al., Deformation of red blood cells, air bubbles, and droplets in microfluidic devices: flow visualizations and measurements, Micromachines 9 (4) (2018) 151.

[10] L.V. Boas, et al., Assessment of the deformability and velocity of healthy and artificially impaired red blood cells in narrow polydimethylsiloxane (PDMS) microchannels, Micromachines 9 (8) (2018) 384.

[11] D. Pinho, et al., Red blood cells radial dispersion in blood flowing through microchannels: The role of temperature, J. Biomech. 49 (11) (2016) 2293-2298.

[12] E. Kaliviotis, J.M. Sherwood, S. Balabani, Partitioning of red blood cell aggregates in bifurcating microscale flows, Sci. Rep. 7 (2017) 44563.

[13] P.K. Ong, et al., Effect of erythrocyte aggregation and flow rate on cell-free layer formation in arterioles, Am. J. Physiol.-Heart Circulatory Physiol. 298 (6) (2010) H1870-H1878.

[14] P.K. Ong, S. Kim, Effect of erythrocyte aggregation on spatiotemporal variations in cell-free layer formation near on arteriolar bifurcation, Microcirculation 20 (5) (2013) 440-453.

[15] D. Katanov, G. Gompper, D.A. Fedosov, Microvascular blood flow resistance: role of red blood cell migration and dispersion, Microvasc. Res. 99 (2015) 57-66.

[16] D. Sampaio, D. Lopes, V. Semiao, Horse and dog blood flows in PDMS rectangular microchannels: experimental characterization of the plasma layer under different flow conditions, Exp. Therm Fluid Sci. 68 (2015) 205-215.

[17] S. Tripathi, et al., Passive blood plasma separation at the microscale: a review of design principles and microdevices, J. Micromech. Microeng. 25 (8) (2015) 083001.

[18] R.O. Rodrigues, et al., In vitro blood flow and cell-free layer in hyperbolic microchannels: Visualizations and measurements, Biochip J. 10 (1) (2016) 9-15. 
[19] D. Bento, et al., Microbubble moving in blood flow in microchannels: effect on the cell-free layer and cell local concentration, Biomed. Microdevices 19 (1) (2017) 6.

[20] D. Pinho, et al., In vitro particulate analogue fluids for experimental studies of rheological and hemorheological behavior of glucose-rich RBC suspensions, Biomicrofluidics 11 (5) (2017) 054105.

[21] D. Bento, et al., Cell-free layer measurements of in vitro blood flow in a microfluidic network: an automatic and manual approach, Comput. Methods Biomech. Biomed. Eng.: Imaging Visualization 6 (6) (2018) 629-637.

[22] J. Chesnutt, J. Marshall, Effect of particle collisions and aggregation on red blood cell passage through a bifurcation, Microvasc. Res. 78 (3) (2009) 301-313.

[23] G. Enden, A. Popel, A numerical study of the shape of the surface separating flow into branches in microvascular bifurcations, J. Biomech. Eng. 114 (3) (1992) 398-405.

[24] J.O. Barber, et al., Simulated two-dimensional red blood cell motion, deformation, and partitioning in microvessel bifurcations, Ann. Biomed. Eng. 36 (10) (2008) $1690-1698$

[25] T. Hyakutake, et al., Numerical study on flows of red blood cells with liposomeencapsulated hemoglobin at microvascular bifurcation, J. Biomech. Eng. 130 (1) (2008) 011014

[26] X. Li, A.S. Popel, G.E. Karniadakis, Blood-plasma separation in Y-shaped bifurcating microfluidic channels: a dissipative particle dynamics simulation study, Phys. Biol. 9 (2) (2012) 026010.

[27] W. Xiong, J. Zhang, Two-dimensional lattice Boltzmann study of red blood cell motion through microvascular bifurcation: cell deformability and suspending viscosity effects, Biomech. Model. Mechanobiol. 11 (3-4) (2012) 575-583.

[28] T. Ye, L. Peng, Y. Li, Three-dimensional motion and deformation of a red blood cell in bifurcated microvessels, J. Appl. Phys. 123 (6) (2018) 064701.

[29] Y.-C. Fung, Stochastic flow in capillary blood vessels, Microvasc. Res. 5 (1) (1973) $34-48$.

[30] A.R. Pries, K. Ley, M. Claassen, P. Gaehtgens, Red cell distribution at microvascular bifurcations, Microvasc. Res. 38 (1989) 81-101.

[31] A.R. Pries, T.W. Secomb, Microvascular blood viscosity in vivo and the endothelial surface layer, Am. J. Physiol.-Heart Circulatory Physiol. 289 (6) (2005) H2657-H2664.

[32] K. Svanes, B. Zweifach, Variations in small blood vessel hematocrits produced in hypothermic rats by micro-occlusion, Microvasc. Res. 1 (2) (1968) 210-220.

[33] P. Cabrales, A.G. Tsai, M. Intaglietta, Increased plasma viscosity prolongs microhemodynamic conditions during small volume resuscitation from hemorrhagic shock, Resuscitation 77 (3) (2008) 379-386.

[34] G. Schmid-Schönbein, et al., Cell distribution in capillary networks, Microvasc. Res. 19 (1) (1980) 18-44.
[35] R.D. Jäggi, R. Sandoz, C.S. Effenhauser, Microfluidic depletion of red blood cells from whole blood in high-aspect-ratio microchannels, Microfluid. Nanofluid. 3 (1) (2007) 47-53.

[36] Kodama, et al., In vitro analysis of blood flow in a microvascular network with realistic geometry, J. Biomech. (2019) under review.

[37] T. Ishikawa, H. Fujiwara, N. Matsuki, T. Yoshimoto, Y. Imai, H. Ueno, T. Yamaguchi, Asymmetry of blood flow and cancer cell adhesion in a microchannel with symmetric bifurcation and confluence, Biomed. Microdevices 13 (2011) 159-167.

[38] V. Leble, et al., Asymmetry of red blood cell motions in a microchannel with a diverging and converging bifurcation, Biomicrofluidics 5 (4) (2011) $44120-4412015$

[39] E. Pinto, et al., A rapid and low-cost nonlithographic method to fabricate biomedical microdevices for blood flow analysis, Micromachines 6 (1) (2015) 121-135.

[40] P.K. Ong, S. Jain, S. Kim, Spatio-temporal variations in cell-free layer formation near bifurcations of small arterioles, Microvasc. Res. 83 (2) (2012) 118-125.

[41] B. Namgung, et al., Near-wall migration dynamics of erythrocytes in vivo: effects of cell deformability and arteriolar bifurcation, Front. Physiol. 8 (2017) 963.

[42] H.H. Lipowsky, Microvascular rheology and hemodynamics, Microcirculation 12 (1) (2005) 5-15.

[43] T.G. Papaioannou, C. Stefanadis, Vascular wall shear stress: basic principles and methods, Hellenic J. Cardiol. 46 (1) (2005) 9-15.

[44] V. Faustino, et al., Biomedical microfluidic devices by using low-cost fabrication techniques: A review, J. Biomech. 49 (11) (2016) 2280-2292.

[45] Y. Fung, Biomechanics: Circulation 2nd. Springer Science, New York, 1997.

[46] M. Abramoff, P. Magelhaes, S. Ram, Image processing with image, Int. J. Biophotonics 11 (7) (2004) 36-42.

[47] N. Tateishi, et al., Flow dynamics of erythrocytes in microvessels of isolated rabbit mesentery: Cell-free layer and flow resistance, J. Biomech. 27 (9) (1994) $1119-1125$.

[48] S. Kim, P.K. Ong, O. Yalcin, M. Intaglietta, P.C. Johnson, The cell-free layer in microvascular blood flow, Biorheology 46 (2009) 181-189.

[49] Valdemar Garcia, R. Dias, R. Lima, In vitro blood flow behaviour in microchannels with simple and complex geometries, in Applied Biological Engineering - Principles and Practice Ganesh R. Naik, Editor, 2012, pp. 393-416.

[50] C.D. Murray, The physiological principle of minimum work applied to the angle of branching of arteries, J. General Physiol. 9 (6) (1926) 835.

[51] A. Kloosterman, et al., Quantification of blood flow and topology in developing vascular networks, PLoS ONE 9 (5) (2014) e96856.

[52] K. Zografos, et al., A design rule for constant depth microfluidic networks for power-law fluids, Microfluid. Nanofluid. 19 (3) (2015) 737-749. 